\title{
INVERSE COEFFICIENT PROBLEMS FOR NONLINEAR ELLIPTIC EQUATIONS
}

\author{
RUNSHENG YANG ${ }^{\boxplus 1}$ and YUNHUA OU ${ }^{2}$
}

(Received 12 June, 2006; revised 3 October, 2006)

\begin{abstract}
This paper is devoted to a class of inverse coefficient problems for nonlinear elliptic equations. The unknown coefficient of the elliptic equations depends on the gradient of the solution and belongs to a set of admissible coefficients. It is shown that the nonlinear elliptic equations are uniquely solvable for the given class of coefficients. Proof of the existence of a quasisolution of the inverse problems is obtained.

2000 Mathematics subject classification: primary 35R30; secondary $35 \mathrm{~J} 85$.

Keywords and phrases: elliptic equations, inverse coefficient problems, existence of quasisolutions.
\end{abstract}

\section{Introduction}

In this paper we consider the problem of determining the unknown coefficient $k(\xi)$ in the nonlinear elliptic boundary value problem

$$
\left\{\begin{aligned}
-\nabla\left(k\left(|\nabla u|^{2}\right) \nabla u\right) & =f(x), & & x \in \Omega \\
u(x) & =0, & & x \in \Gamma_{1}, \Gamma_{1} \subset \Gamma=\partial \Omega \\
k\left(|\nabla u|^{2}\right) \frac{\partial u}{\partial n} & =\varphi(x), & & x \in \Gamma_{2},
\end{aligned}\right.
$$

from knowledge of the measured data given on the boundary

$$
u(x)=g(x), \quad x \in \Gamma_{2},
$$

where the domain $\Omega \subset R^{N}(N \geq 1)$ is assumed to be bounded and simply connected with a piecewise smooth boundary $\Gamma$ and $\Gamma_{1} \cap \Gamma_{2}=\emptyset, \bar{\Gamma}_{1} \cup \bar{\Gamma}_{2}=\Gamma$, meas $\Gamma_{1} \neq 0$.

\footnotetext{
'Department of Mathematics, Changsha University of Sciences and Technology, Changsha, Hunan 410076, P. R. China; email: runshengyang@126.com.

${ }^{2}$ Department of Mathematics, Hunan University of Technology, Zhuzhou, Hunan 412007, P. R. China; email: yunhuaou01@126.com.

(C) Australian Mathematical Society 2007, Serial-fee code 1446-1811/07
} 
The determination of unknown coefficients in elliptic partial differential equations from additional boundary conditions (measured data taken on the boundary) is known in the literature as inverse coefficient problems (ICPs). Physically, an ICP is the reconstruction of an intraproperty of a medium (for example, conductivity or permittivity) in some bounded region by using steady-state measurements on the boundary.

ICPs for linear elliptic equations have been studied by many people, for example, by Cannon and DuChateau [1] for rectangular domains, and by Cannon and Rundell [2] for unbounded domains. ICPs for semilinear parabolic equations have also been considered by Liu [8-12]. In the case of elliptic equations, uniqueness and continuous dependence results for various inverse problems have been proved by Kohn and Vogelius [6] and Sylvester and UhImann [13].

For a given coefficient $k=k(s)$, we sometimes call the problem (1.1) the direct problem DP. Denote the solution of DP by $u[x ; k]$. Then from the additional condition (1.2), we see that the ICP (1.2) consists of solving the nonlinear functional equation

$$
u[x ; k]=g(x), \quad x \in \Gamma_{2}
$$

for given data $g=g(x)$, over the solution $u=u[x ; k]$ of the nonlinear boundary value problem (1.1).

In applications, instead of the measured data (1.2) on the boundary, one may get the nonlocal measured data

$$
\int_{\Omega} u(x) d x=\Phi
$$

In this case we shall define the problem of determining a solution $k=k(s)$ of the nonlinear functional equation

$$
\int_{\Omega} u[x ; k] d x=\Phi
$$

over the solution of the DP as a nonlocal inverse coefficient problem.

In the practical solution of such ICPs, instead of solving the functional equations above, one usually tries to find the solutions of the minimization problem

$$
I_{1}(k)=\min _{\tilde{k} \in K} I_{1}(\tilde{k}), \quad \text { where } \quad I_{1}(\tilde{k})=\int_{\Gamma_{2}}|u[x ; \tilde{k}]-g(x)|^{2} d x
$$

is an auxiliary functional and $K$ is a set of admissible coefficients. Tikhonov and Arsenin [14] call a solution of the minimization problem (1.4) a quasisolution of the ICP (1.2) (see, for example, [3-5] for more detail).

For the ICP (1.3), a quasisolution can be defined as a solution of the minimization problem

$$
I_{2}(k)=\min _{\tilde{k} \in K} I_{2}(\tilde{k}), \quad \text { where } \quad I_{2}(\tilde{k})=\left|\int_{\Omega} u[x ; \tilde{k}] d x-\Phi\right|
$$


The ICPs (1.2) and (1.3) have been considered by Hasanov [4] under the set $\mathbf{K}_{\varepsilon}$ of coefficients $k(s)$ satisfying the following conditions:

(a) $0<c_{1} \leq k(s) \leq c_{2}$

(b) $k^{\prime}(s) \leq 0$

(c) $k(s)+2 k^{\prime}(s) s \geq c_{3}>0, s \in\left(0, s^{*}\right)$

(d) $\exists s_{0}, k(s)+2 k^{\prime}(s) s_{0} \geq c_{3}>0, s \in\left(0, s_{0}\right)$

(e) $k^{\prime}(s)$ is a monotone increasing (or decreasing) function on $\left(0, s^{*}\right)$.

In the set of admissible coefficients $\mathbf{K}_{\varepsilon}$, Hasanov [4] has proved that the ICP (1.2) has at least one quasisolution.

However, in many applications, the coefficient $k(s)$ does not satisfy the conditions above. It may not be monotone and piecewise smooth. Also, it is not easy to estimate the constant $s^{*}$, which should be an a priori upper bound for $|\nabla u(x)|^{2}, \forall x \in \Omega$. Therefore, one should consider the ICPs in the interval $[0, \infty)$ instead of in $\left[0, s^{*}\right]$. In the following we shall generalize the results in [4].

With respect to the coefficients $k=k(s)$ we make the following assumptions:

(A1) $k \in C[[0, \infty)]$ and $c_{1} \leq k(s) \leq c_{2}, \forall s \in[0, \infty)$;

(A2) $\sum_{i=1}^{N}\left[k\left(|\xi|^{2}\right) \xi_{i}-k\left(\left|\xi^{\prime}\right|^{2}\right) \xi_{i}^{\prime}\right]\left(\xi_{i}-\xi_{i}^{\prime}\right) \geq c_{3}\left|\xi-\xi^{\prime}\right|^{2} \forall \xi, \xi^{\prime} \in R^{N}$.

Here $c_{1}, c_{2}$ and $c_{3}$ are positive constants.

The set $K$ of coefficients satisfying Assumptions (A1) and (A2) is called the set of admissible coefficients for the ICPs under consideration.

Comparing with the conditions (a)-(e), we have the following result.

LEMMA 1.1. Suppose that a coefficient $k(s)$ satisfies the conditions (a)-(c) above with $s^{*}=\infty$. Then $k \in K$.

PROOF. Let the coefficient $k(s)$ satisfy the conditions (a)-(c). By the definition of the admissible coefficient set $K$, we have to show that $k$ satisfies Assumption (A2). For all $\xi, \xi^{\prime} \in R^{N}$ and $t \in[0,1]$, define the functions

$$
\Psi(t)=t \xi+(1-t) \xi^{\prime} \quad \text { and } \quad f(t)=k\left(|\Psi(t)|^{2}\right)\left[\Psi(t)\left(\xi-\xi^{\prime}\right)\right]
$$

Then

$$
\begin{aligned}
& \sum_{i=1}^{N}\left[k\left(|\xi|^{2}\right) \xi_{i}-k\left(\left|\xi^{\prime}\right|^{2}\right) \xi_{i}^{\prime}\right]\left(\xi_{i}-\xi_{i}^{\prime}\right) \\
& \quad=k\left(|\xi|^{2}\right) \xi\left(\xi-\xi^{\prime}\right)-k\left(\left|\xi^{\prime}\right|^{2}\right) \xi^{\prime}\left(\xi-\xi^{\prime}\right) \\
& \quad=f(1)-f(0)=\int_{0}^{1} f^{\prime}(t) d t \\
& \quad=\int_{0}^{1} k\left(|\Psi(t)|^{2}\right) \cdot\left|\xi-\xi^{\prime}\right|^{2}+2 k^{\prime}\left(|\Psi(t)|^{2}\right)\left[\Psi(t)\left(\xi-\xi^{\prime}\right)\right]^{2} d t
\end{aligned}
$$




$$
\begin{aligned}
& \geq \int_{0}^{1}\left[k\left(|\Psi(t)|^{2}\right)+2 k^{\prime}\left(|\Psi(t)|^{2}\right)|\Psi(t)|^{2}\right]\left|\xi-\xi^{\prime}\right|^{2} d t \\
& \geq c_{3}\left|\xi-\xi^{\prime}\right|^{2} .
\end{aligned}
$$

The first and second inequalities above use the Cauchy inequality, and conditions (b) and (c), respectively. The proof is now complete.

Let $V=\left\{v \in H^{1}(\Omega): \gamma v=0\right.$ on $\left.\Gamma_{1}\right\}$ and $H=L^{2}(\Omega)$, where $\gamma: H^{1}(\Omega) \rightarrow$ $L^{2}(\partial \Omega)$ denotes the trace operator. Applying the Poincaré inequality we may define the Hilbert space $V$ with norm $\|u\|_{V}=\left(\int_{\Omega}|\nabla u|^{2} d x\right)^{1 / 2}$. Identifying $H$ with its dual, we have an evolution triple $V \subset H \subset V^{*}$ with dense, continuous and compact embeddings (see, for example, Zeidler [15]). For convenience, we denote by $(\cdot, \cdot\rangle_{B}$ the duality of $B$ and its dual $B^{*}$ as well as the norm by $\|\cdot\|_{B}$ for any Banach space $B$.

For any $k \in K$, we define the nonlinear operator $A: V \rightarrow V^{*}$ by

$$
\langle A u, v\rangle_{V}=\int_{\Omega} k\left(|\nabla u|^{2}\right) \nabla u \nabla v d x \quad \forall u, v \in V .
$$

Since we always assume that $f \in H$ and $\varphi \in L^{2}\left(\Gamma_{2}\right)$, the linear functional $F$ on $V$ is well defined by

$$
\langle F, v\rangle_{V}=\int_{\Omega} f(x) v(x) d x+\int_{\Gamma_{2}} \varphi(x) v(x) d x
$$

Using the operators defined above, it is easy to see that a weak solution of the problem DP can be defined as a solution of the variational problem

$$
\langle A u, v\rangle_{v}=\langle F, v\rangle_{v} \quad \forall v \in V
$$

\section{The inverse coefficient problem}

The first theorem we intend to prove is as follows.

THEOREM 2.1. If $k \in K$, the problem $D P$ has a unique solution $u \in V$. If $u$ is the solution to DP, then there exists a constant $c>0$ (independent of $k \in K$ ) such that

$$
\|u\|_{v} \leq c\left(\|f\|_{H}+\|\varphi\|_{L^{2}\left(\Gamma_{2}\right)}\right)
$$

ProOF. Applying a well-known existence theorem for monotone operators (see, for example, Zeidler [15]), we readily obtain that DP has a unique solution in $V$ for any $k \in K$. 
Let $u$ be the solution to DP. We notice that $u \in V$. Using (1.1) and integration by parts, we have

$$
\int_{\Omega} k\left(|\nabla u|^{2}\right)|\nabla u|^{2} d x=\int_{\Omega} f u d x+\int_{\Gamma_{2}} \varphi(\gamma u) d x .
$$

By Assumption (A1), it follows that

$$
c_{1} \int_{\Omega}|\nabla u|^{2} d x \leq \int_{\Omega} k\left(|\nabla u|^{2}\right)|\nabla u|^{2} d x
$$

Moreover, using the Young inequality with any $\epsilon>0$, we have

$$
\int_{\Omega} f u d x \leq \frac{\epsilon^{2}}{2} \int_{\Omega} u^{2} d x+\frac{1}{2 \varepsilon^{2}}\|f\|_{H}^{2} \leq \frac{\epsilon^{2} c_{0}^{2}}{2} \int_{\Omega}|\nabla u|^{2} d x+\frac{1}{2 \varepsilon^{2}}\|f\|_{H}^{2} .
$$

In the last inequality, we have used the Poincaré inequality, where $c_{0}$ is the Poincaré constant.

By virtue of the boundedness of the trace operator $\gamma$ and the Young inequality, we obtain

$$
\begin{aligned}
\int_{\partial \Omega} \varphi(\gamma u) d x & \leq \frac{\epsilon^{2}}{2} \int_{\partial \Omega}|\gamma u|^{2} d x+\frac{1}{2 \varepsilon^{2}}\|\varphi\|_{L^{2}\left(\Gamma_{2}\right)}^{2} \\
& \leq \frac{\epsilon^{2}\|\gamma\|^{2}}{2} \int_{\Omega}|\nabla u|^{2} d x+\frac{1}{2 \varepsilon^{2}}\|\varphi\|_{L^{2}\left(\Gamma_{2}\right)}^{2} .
\end{aligned}
$$

From the above bounds and (2.2), we may choose $\epsilon>0$ small enough and readily deduce (2.1). This completes the proof.

In what follows we analyze the class of admissible coefficients and prove stability of the coefficient and then obtain the main result - the existence theorem for the inverse problem. As seen above, the two Assumptions (A1) and (A2) guarantee the solvability of the nonlinear DP in $V$. Therefore, when defining a set of admissible coefficients for the ICPs under consideration, some conditions have already been given. On the other hand, it is natural to endeavour to obtain a solution of any ICP with minimal requirements on the desired coefficient. Unfortunately, in many cases the given conditions (physical or mathematical, such as the DP solvability conditions (A1) and (A2)) do not guarantee the compactness of the set of admissible coefficients in the suitable space. Therefore, the main problem is to construct a compact set of admissible coefficients with minimal additional conditions with respect to $k=k(s)$.

Now we turn to the solvability of inverse coefficient problems. In order to obtain a theorem on the existence of quasisolutions for ICPs, we need the following result.

THEOREM 2.2. Suppose that a sequence of coefficients $k_{m} \in K$ converges to a function $k \in K$ in the following way:

$$
\lim _{m \rightarrow \infty}\left|k_{m}(s)-k(s)\right|=0, \quad \forall s \in[0, \infty)
$$


Then the sequence of solutions $u_{m}=u\left(x ; k_{m}\right)$ converges to the solution $u=u(x ; k)$ in $V$.

PROOF. Since the sequence $k, k_{m} \in K(m=1,2, \ldots)$, by Theorem 2.1 the solutions $u, u_{m}(m=1,2,3, \ldots)$ are well defined. By the definition of solutions for DP, we have $\forall v \in V$

$$
\begin{aligned}
\int_{\Omega} k_{m}\left(\left|\nabla u_{m}\right|^{2}\right) \nabla u_{m} \nabla v d x & =\int_{\Omega} f v d x+\int_{\Gamma_{2}} \varphi(\gamma v) d x, \\
\int_{\Omega} k\left(|\nabla u|^{2}\right) \nabla u \nabla v d x & =\int_{\Omega} f v d x d t+\int_{\Gamma_{2}} \varphi(\gamma v) d x,
\end{aligned}
$$

which imply

$$
\int_{\Omega}\left[k_{m}\left(\left|\nabla u_{m}\right|^{2}\right) \nabla u_{m}-k\left(|\nabla u|^{2}\right) \nabla u\right] \nabla\left(u_{m}-u\right) d x=0 .
$$

Therefore, we get

$$
\begin{aligned}
& \int_{\Omega}\left[k_{m}\left(\left|\nabla u_{m}\right|^{2}\right) \nabla u_{m}-k_{m}\left(|\nabla u|^{2}\right) \nabla u\right] \nabla\left(u_{m}-u\right) d x \\
& \quad+\int_{\Omega}\left[k_{m}\left(|\nabla u|^{2}\right)-k\left(|\nabla u|^{2}\right)\right] \nabla u \nabla\left(u_{m}-u\right) d x=0 .
\end{aligned}
$$

By Assumption (A2) and the Hölder inequality, we obtain

$$
\begin{aligned}
& c_{3} \int_{\Omega}\left|\nabla\left(u_{m}-u\right)\right|^{2} d x \\
& \quad \leq \int_{\Omega}\left|\left[k_{m}\left(|\nabla u|^{2}\right)-k\left(|\nabla u|^{2}\right)\right] \nabla u \nabla\left(u_{m}-u\right)\right| d x \\
& \quad \leq\left\{\int_{\Omega}\left|k_{m}\left(|\nabla u|^{2}\right)-k\left(|\nabla u|^{2}\right)\right|^{2}|\nabla u|^{2} d x\right\}^{1 / 2}\left\{\int_{\Omega}\left|\nabla\left(u_{m}-u\right)\right|^{2} d x\right\}^{1 / 2},
\end{aligned}
$$

which implies that

$$
c_{3}^{2} \int_{\Omega}\left|\nabla\left(u_{m}-u\right)\right|^{2} d x \leq \int_{\Omega}\left|k_{m}\left(|\nabla u|^{2}\right)-k\left(|\nabla u|^{2}\right)\right|^{2}|\nabla u|^{2} d x .
$$

By Assumption (Al), we have

$$
\left|k_{m}\left(|\nabla u|^{2}\right)-k\left(|\nabla u|^{2}\right)\right|^{2}|\nabla u|^{2} \leq c_{2}^{2}|\nabla u|^{2}
$$

By virtue of (2.3), (2.5) and Lebesgue's convergence theorem, we obtain

$$
\lim _{m \rightarrow \infty} \int_{\Omega}\left|k_{m}\left(|\nabla u|^{2}\right)-k\left(|\nabla u|^{2}\right)\right|^{2}|\nabla u|^{2} d x=0
$$

Therefore, using inequality (2.4), it is easy to see that

$$
\lim _{m \rightarrow \infty}\left\|u_{m}-u\right\|_{V}=0 .
$$

The proof of the theorem is complete. 
Next we study the existence of a quasisolution of the ICPs (1.2) and (1.3). For this reason we need a compact set of coefficients and continuity of the functionals $I_{1}(k)$ and $I_{2}(k)$ defined in the previous section, respectively. First we note that the two Assumptions (Al) and (A2) that compose the set of admissible coefficients $K$ arise as solvability conditions for the problem DP. By virtue of Theorem 2.2, it is natural to construct a compact set of admissible coefficients in $C[[0, \infty)]$. For this reason, in addition to Assumptions (A1) and (A2), we assume that the subset $K_{c}$ of $K$ is equicontinuous, that is, $K_{c} \subset K$ and for every $\epsilon>0$, there exists $\delta>0$ such that if $k \in K_{c}, s_{1}, s_{2} \in[0, \infty)$ and $\left|s_{1}-s_{2}\right|<\delta$, then $\left|k\left(s_{1}\right)-k\left(s_{2}\right)\right|<\epsilon$.

Now we shall show the following generalized Ascoli-Arzela theorem.

THEOREM 2.3. Let $K_{c}$ be an equicontinuous subset of $K$. Then for any sequence $\left\{k_{m}\right\}$ of coefficients in $K_{c}$, there exists a subsequence, still denoted by $\left\{k_{m}\right\}$, such that $\lim _{m \rightarrow \infty} k_{m}(s)=k(s), \forall s \in[0, \infty)$ and $k \in K_{c}$.

PROOF. The idea of the proof is similar to that of the Ascoli-Arzela theorem. For the convenience of the reader, we give an outline here.

It is well known that the interval $[0, \infty)$ is separable. One may choose a dense countable subset $\left\{s_{i}\right\}$ in it. By use of Assumption (A1), there is a subsequence $\left\{k_{m}^{(1)}\right\} \subseteq\left\{k_{m}\right\}$ such that the sequence $\left\{k_{m}^{(1)}\left(s_{1}\right)\right\}$ converges to a real number, denoted by $k\left(s_{1}\right)$. Then by Assumption (A 1$)$ again, one may choose a subsequence $\left\{k_{m}^{(2)}\right\} \subseteq$ $\left\{k_{m}^{(1)}\right\}$ such that the sequence $\left\{k_{m}^{(2)}\left(s_{2}\right)\right\}$ converges to a real number, denoted by $k\left(s_{2}\right)$. Repeating the procedure, we obtain a subsequence $\left\{k_{m}^{(n)}\right\}$ such that

$$
\lim _{m \rightarrow \infty} k_{m}^{(n)}\left(s_{i}\right)=k\left(s_{i}\right), \quad i=1,2, \ldots, n .
$$

We easily conclude that the subsequence $\left\{k_{m}^{(m)}\right\} \subseteq\left\{k_{m}\right\}$ constructed above has

$$
\lim _{m \rightarrow \infty} k_{m}^{(m)}\left(s_{i}\right)=k\left(s_{i}\right), \quad i=1,2,3, \ldots
$$

Furthermore, we shall prove that $k_{m}^{(m)}(s)$ is a convergent sequence for all $s \in[0, \infty)$. By use of the equicontinuity, for every $\epsilon>0$, there exists $\delta>0$ such that if $s, s^{\prime} \in[0, \infty)$ and $\left|s-s^{\prime}\right|<\delta$, then $\left|k_{m}^{(m)}(s)-k_{m}^{(m)}\left(s^{\prime}\right)\right|<\epsilon / 3, m=1,2,3, \ldots$ Since $\left\{s_{i}\right\}$ is dense in $[0, \infty), \forall s \in[0, \infty) \backslash\left\{s_{i}\right\}$, we can get an $s_{i_{0}} \in\left\{s_{i}\right\}$ such that $\left|s_{i_{0}}-s\right|<\delta$ and by the convergence of $\left\{k_{m}^{(m)}\left(s_{i_{0}}\right)\right\}$, one can choose $M>0$ such that $\left|k_{m}^{(m)}\left(s_{i_{0}}\right)-k_{m^{\prime}}^{\left(m^{\prime}\right)}\left(s_{i_{0}}\right)\right|<\epsilon / 3, \forall m, m^{\prime} \geq M$. By the inequalities above, if $m, m^{\prime} \geq M$, we easily get

$$
\begin{aligned}
\left|k_{m}^{(m)}(s)-k_{m^{\prime}}^{\left(m^{\prime}\right)}(s)\right| \leq & \left|k_{m}^{(m)}(s)-k_{m}^{(m)}\left(s_{i_{0}}\right)\right|+\left|k_{m}^{(m)}\left(s_{i_{0}}\right)-k_{m^{\prime}}^{\left(m^{\prime}\right)}\left(s_{i_{0}}\right)\right| \\
& +\left|k_{m^{\prime}}^{\left(m^{\prime}\right)}\left(s_{i_{0}}\right)-k_{m^{\prime}}^{\left(m^{\prime}\right)}(s)\right|<\frac{\epsilon}{3}+\frac{\epsilon}{3}+\frac{\epsilon}{3}=\epsilon,
\end{aligned}
$$


which implies that $k_{m}^{m}(s)$ is a Cauchy sequence. Therefore, we have shown that

$$
\lim _{m \rightarrow \infty} k_{m}^{(m)}(s)=k(s) \quad \forall s \in[0, \infty) .
$$

Similarly we easily conclude that $k \in K_{c}$. The proof is complete.

REMARK. Let $K_{h}$ be a uniformly Hölder continuous subset of $K$. Then $K_{h}$ is equicontinuous. In particular, any subsets of $K$ which are bounded in $H^{1}([0, \infty))$ are equicontinuous.

Using the compactness of the class of admissible cnefficients $K_{c} \subset K$, we can prove the following existence theorem for ICPs.

THEOREM 2.4. Both ICPs (1.2) and (1.3) have at least one quasisolution in the set of admissible coefficients $K_{c}$.

PROOF. Let $\left\{k_{m}\right\} \subset K_{c}$ be a minimizing sequence of the functional $I_{2}$ on $K_{c}$ defined by (1.4). By Theorem 2.3, we may assume that $k_{m}(s) \rightarrow k(s)$ as $m \rightarrow \infty, \forall s \epsilon$ $[0, \infty)$. Using Theorem 2.2, the sequence $u_{m}=u\left(x ; k_{m}\right)$ converges to $u=u(x ; k)$ in $V$. Applying the trace theorem (see [7, Theorem 6.5]), we conclude that the sequence $\left\{u_{m}\right\}$ converges to $u$ in $L^{2}\left(\Gamma_{2}\right)$. Therefore, we have

$$
\min _{\tilde{k} \in K_{c}} I_{1}(\tilde{k})=\lim _{m \rightarrow \infty} I_{1}\left(k_{m}\right)=I_{1}(k) .
$$

Similarly, we can show the existence of quasisolutions of $I_{2}$ on $K_{c}$. The proof is complete.

COROLLARY 2.5. Let $K_{o}$ be a subset of admissible coefficients satisfying the conditions (a)-(d) with $s^{*}=\infty$. Then both ICPs (1.2) and (1.3) have at least one quasisolution in $K_{0}$.

PROOF. By Lemma 1.1, we get that $K_{0} \subseteq K$. It is easy to see from (a) and (d) that $\forall k \in K_{0}, \forall s \in[0, \infty), c_{3}-c_{2} / 2 s_{0} \leq k^{\prime}(s) \leq 0$. Therefore, $K_{0}$ is an equicontinuous subset of $K$. From this point, we can use the same argument as that in Theorem 2.4 to show the corollary. The proof is complete.

\section{Acknowledgements}

The authors thank the referee for comments and suggestions on the original manuscript which led to its improvement. 
This project was supported by the NNSF of China under Grant No.10671211 and the NSF of Hunan Province under Grant No.07JJ3005.

\section{References}

[1] J. R. Cannon and P. C. DuChateau, "Determination of the conductivity of an isotropic medium", J. Math. Anal. Appl. 48 (1974) 699-707.

[2] J. R. Cannon and W. Rundell, "An inverse problem for an elliptic partial differential equation", $J$. Math. Anal. Appl. 126 (1987) 329-340.

[3] A. Hasanov, "An inverse coefficient problem for an elasto-plastic medium", SIAM J. Appl. Math. 55 (1995) 1736-1752.

[4] A. Hasanov, "Inverse coefficient problems for monotone potential operators", Inverse Problems 13 (1997) 1265-1278.

[5] A. Hasanov, "Inverse coefficient problems for elliptic variational inequalities with a nonlinear operator", Inverse Problems 14 (1998) 1251-1269.

[6] R. Kohn and M. Vogelius, "Determining conductivity by boundary measurements", Commun. Pure Appl. Math. 37 (1984) 289-298.

[7] O. A. Ladyzhenskaya, Boundary value problems in mathematical physics (Springer, New York, 1985).

[8] Zhenhai Liu, "On the identification of coefficients of semilinear parabolic equations", Acta Math. A.ppl. Sinica 10 (1994) 356-367.

[9] Zhenhai Liu, "A class of evolution hemivariational inequalities", Nonlinear Anal. 36 (1999) 91100.

[10] Zhenhai Liu, "Identification of parameters in semilinear parabolic equations", Acta Math. Sci. 19 (1999) 175-180.

[11] Zhenhai Liu, "Browder-Tikhonov regularization of non-coercive evolution hemivariational inequalities", Inverse Problems 21 (2005) 13-20.

[12] Zhenhai Liu, "Strong convergence results for hemivariational inequalities", Sci. China Ser. A 49 (2006) 893-901.

[13] J. Sylvester and G. Uhlmann, "Inverse boundary value problems of the boundary-continuous dependence", Commun. Pure Appl. Math. 41 (1988) 197-219.

[14] A. Tikhonov and V. Arsenin, Solutions of ill-posed problems (Wiley, New York, 1977).

[15] E. Zeidler, Nonlinear functional analysis and its applications II A/B (Springer, New York, 1990). 